Dinh, Christoph; Strohmeier, Daniel; Haueisen, Jens; Güllmar, Daniel:

Brain atlas based region of interest selection for real-time source localization using k-means lead field clustering and RAP-MUSIC

Zuerst erschienen in: $\quad$ Biomedical Engineering = Biomedizinische Technik. - Berlin [u.a.] : de Gruyter. - 57 (2012), Suppl. 1, Track-O, p. 813.

Erstveröffentlichung: 2012-09-06

ISSN (online): $\quad$ 1862-278X

ISSN (print): $\quad$ 0013-5585

DOI: $\quad 10.1515 / \mathrm{bmt}-2012-4316$

[Zuletzt gesehen: 2019-08-15]

„Im Rahmen der hochschulweiten Open-Access-Strategie für die Zweitveröffentlichung identifiziert durch die Universitätsbibliothek IImenau."

"Within the academic Open Access Strategy identified for deposition by Ilmenau University Library."

„Dieser Beitrag ist mit Zustimmung des Rechteinhabers aufgrund einer (DFGgeförderten) Allianz- bzw. Nationallizenz frei zugänglich."

"This publication is with permission of the rights owner freely accessible due to an Alliance licence and a national licence (funded by the DFG, German

DFG

Research Foundation) respectively." 


\section{Brain Atlas based Region of Interest Selection for Real-Time Source Localization using K-Means Lead Field Clustering and RAP-MUSIC}

C. Dinh, Institute of Biomedical Engineering and Informatics, Ilmenau University of Technology, Ilmenau, Germany, christoph.dinh@tu-ilmenau.de

D. Strohmeier, Institute of Biomedical Engineering and Informatics, Ilmenau University of Technology, Germany, daniel.strohmeier@tu-ilmenau.de

J. Haueisen, Institute of Biomedical Engineering and Informatics, Ilmenau University of Technology, Germany, jens.haueisen@tu-ilmenau.de

D. Güllmar, Department of Radiology, Jena University Hospital, Jena, Germany, daniel.guellmar@med.uni-jena.de

\section{Introduction}

Real-Time source localization from single trial electroencephalography (EEG) / magnetoencephalography (MEG) has recently been brought into focus of neuroscience to clarify brain functionality, identify mental states and to improve online applications such as brain-computer interface systems. To succeed with real-time single trial localization two major challenges have to be solved: first, handling the low signal-to-noise ratio (SNR) and second, managing the computational effort. We aim to address both by reducing the lead field through clustering the lead field dipoles.

\section{Methods}

With low SNR the possible source localization resolution of single trial measurements is much lower compared to averaged signals, meaning, it is not possible to distinguish as many sources. Due to that circumstance we are clustering similar lead field dipoles, which we are likely not able to differentiate by a localization algorithm. In order to maintain the position information of the resulting clusters, the clustering is done region of interest (ROI) wise. The ROIs are defined by the Destrieux atlas. To keep a high variance we cluster the lead field dipoles of each sensor separately. The clustering is accomplished using a k-means approach, which encapsulates a ROI in $\mathrm{n}$ clusters depending on their size. To localize correlated activated ROIs we are using the RAP-MUSIC scanning approach.

\section{Results}

The localization shows a high accuracy up to a SNR of -20dB in simulations, e.g. the correct or neighbored ROI is localized. We use cluster sizes depending on the given SNR, which results in lead field reductions of 40 to 80 times. This leads to a large amount of calculation steps saved, e.g. a lead field reduction from 8195 to 174 dipoles provides savings by 2206 times.

\section{Conclusion}

This new method allows real-time ROI localization with a computationally intensive scanning approach. In a second processing step a high-resolution localization based on selected ROIs can be applied. 